\author{
S.K. Tleukenov ${ }^{1}$, M.K. Zhukenov ${ }^{2}$, N.A. Ispulov ${ }^{2}$ \\ ${ }^{1}$ L.N. Gumilyov Eurasian National University, Nur-Sultan, Kazakhstan; \\ ${ }^{2}$ S. Toraighyrov Pavlodar State University, Kazakhstan \\ (E-mail: marat_k_zhukenov@mail.ru)
}

\title{
Propagation of electromagnetic waves in anisotropic magnetoelectric medium
}

\begin{abstract}
In the article we consider porpagation and interaction of electromagnetic waves of different polarization, in anisotropic medium that is inhomogeneous along the $\mathrm{Z}$ axis with magnetoelectric effect of tetragonal, trigonal, and hexagonal symmetries are described by the structure of the matrix coefficients. The matricant structure of the original system of equations follows from the structure of coefficient matrix. In unlimited periodic structures, dispersion relations of electromagnetic waves are determined from the new modified conditions for the existence of non-trivial solutions which are the consequence of the matricant structure. Obvious analitical form of the matricants for the homogeneous anisotropic dielectric medium with magnetoelectric effects follows from the matricant structure. Analytical equations for homogeneous anisotropic medium with magnetoelectric effects allow one, in matrix setting, to obtain analytical solutions for the problem of reflection and refraction on the border of isotropic and anisotropic medium with magnetoelectric effect based on the matricant method. Initial relationships that describe electromagnetic wave propagation in anisotropic magnetoelectric medium are reduced to the system of linear homogeneous first order differential equations. The structure of the matricant is obtained. Dispersion equations of electromagnetic waves in periodic inhomogeneous medium with magnetoelectric effects are constructed. Averaged matricant describing the propagation of electromagnetic waves in homogeneous anisotropic medium with magnetoelectric medium are also constructed. Besides, the graphs of energy reflection coefficient of TE and TM electromagnetic waves and incident angle are plotted.
\end{abstract}

Keywords: anisotropic medium; electromagnetic waves; magnetoelectric effect; matricant method; reflection, refraction of electromagnetic waves.

\section{Introduction}

Anisotropic medium is characterized by many parameters. One of the constructive ways to overcome these difficulties is a systematic and detailed study of properties of Maxwell equations in a wide class of anisotropic medium so that the regularities of these solutions that depend on the structure of tensor quantities defining anisotropy of medium can be established. In this research, solutions of Maxwell equations in dielectric magnetoelectric medium that depend on time harmonically are considered [1].

In this work on the basis of a method of variables separation and representation of a solution in the form of plane harmonious waves of Maxwell equation and the defining ratios describing distribution of electromagnetic waves in non-isotropic mediums with magnetoelectric effect are brought to the equivalent system of ordinary differential equations of the 1st order with float factors and matrixes of coefficients for tetragonal, trigonal and hexagonal singoniya in volume and flat cases are received [2]. The structure of matrixes of fundamental solutions of a system of the differential equations of the 1st order describing distribution of electromagnetic waves in anisotropic environments of tetragonal, trigonal and hexagonal singoniya with magnetoelectric effect in volume and flat cases is constructed [3]. The equations of dispersion of electromagnetic waves in unlimited periodic structures are received. Matriciants of homogeneous anisotropic dielectric environments with magnetoelectric effect are constructed. Matrix statement is formulated and the analytical solution of a problem of reflection and refraction of electromagnetic waves on border of the isotropic environment and anisotropic environment with magnetoelectric effect is received [4]. The numerical analysis of power coefficients of reflection and refraction at reflection of electromagnetic waves on border of the isotropic environment and anisotropic environment with magnetoelectric effect is carried out. Schedules of dependence of power coefficients of reflection and refraction from a hade of electromagnetic waves are constructed $[5,6]$. The value of work is that the structure of fundamental decisions and an obvious type of analytical representations of a matriciant allows to investigate periodically non-uniform anisotropic environments and average structures $[7,8]$.

When volume charge density, $\rho$, current density vectors and harmonic time dependance of wave fields are absent Maxwell equations take following form: 


$$
\begin{gathered}
\operatorname{rot} \vec{E}=-\frac{\partial \vec{B}}{\partial t}=-i \omega \vec{B}, \operatorname{rot} \vec{H}=\frac{\partial \vec{D}}{\partial t}=i \omega \vec{D}, \\
\operatorname{div} \vec{B}=0, \operatorname{div} \vec{D}=0 .
\end{gathered}
$$

Material equations connecting $\vec{B}$ and $\vec{H}, \vec{D}$ and $\vec{E}$ we obtain from free energy

$$
F_{\text {эм }}=\varepsilon_{0} \varepsilon_{i j} E_{i} E_{j}+\mu_{0} \mu_{i j} H_{i} H_{j}-\alpha_{i j} E_{i} H_{j},
$$

where $\varepsilon_{i j}, \mu_{i j}$ - components of dielectric and magnetic susceptibility tensors; $\alpha_{i j}$ - component of nonsymmetric magnetoelectric effect tensor.

Solutions of $\vec{E}, \vec{H}, \vec{B}, \vec{D}$ wave fields are taken in the following form:

$$
\vec{F}=\vec{F}(z) e^{i \omega t \pm i k_{x} x \pm i k_{y} y},
$$

where $\omega$ - frequency; $k_{x}, k_{y}$ - components of a wave vector. We assume that medium is inhomogeneous along the $z$ axis. Then material equations take the following form:

$$
\frac{\partial F}{\partial E_{i}}=\varepsilon_{0} \varepsilon_{i j} E_{j}-\alpha_{i j} H_{j}=D_{i} ; \frac{\partial F}{\partial H_{i}}=\mu_{0} \mu_{i j} H_{i}-\alpha_{i j} E_{i}=B_{j} .
$$

Based on the matricant method, the system of equations describing propagation of electromagnetic waves can be reduced to equivalent system of differential equations:

$$
\frac{d \vec{U}}{d z}=B \vec{U}, \vec{U}=\left(E_{y}, H_{x}, H_{y}, E_{x}\right) .
$$

Then matrix coefficients of $\hat{B}$ takes the following form:

where

$$
\widehat{B}=\left(\begin{array}{cccc}
b_{11} & b_{12} & b_{13} & b_{14} \\
b_{21} & b_{11} & b_{23} & b_{24} \\
-b_{24} & -b_{14} & -b_{11} & b_{34} \\
-b_{23} & -b_{13} & b_{43} & -b_{11}
\end{array}\right),
$$

$$
\begin{aligned}
& b_{11}=i \frac{k_{x} k_{y}}{\beta} \alpha_{11} ; \quad b_{12}=i \mu_{0}\left(\frac{k_{y}^{2}}{\beta} \mu_{2}+\omega \mu_{1}\right) ; \quad b_{13}=-i \frac{k_{x} k_{y}}{\beta} \mu_{0} \mu_{2} ; \\
& b_{14}=-i\left(\frac{k_{y}^{2}}{\beta} \alpha_{11}+\omega \alpha_{\perp}\right) ; \quad b_{21}=i \varepsilon_{0}\left(\frac{k_{x}^{2}}{\beta} \varepsilon_{2}+\omega \varepsilon_{1}\right) ; \quad b_{23}=-i\left(\frac{k_{x}^{2}}{\beta} \alpha_{11}+\omega \alpha_{\perp}\right) ; \\
& b_{24}=-i \frac{k_{x} k_{y}}{\beta} \varepsilon_{0} \varepsilon_{2} ; \quad b_{34}=-i \varepsilon_{0}\left(\frac{k_{y}^{2}}{\beta} \varepsilon_{2}+\omega \varepsilon_{1}\right) ; \quad b_{43}=-i \mu_{0}\left(\frac{k_{x}^{2}}{\beta} \mu_{2}+\omega \mu_{1}\right) .
\end{aligned}
$$

Propagation of waves in the $(x z, y z)$ planes is described by $\hat{B}$ matrix:

$$
\hat{B}=\left(\begin{array}{cccc}
0 & b_{12} & 0 & b_{14} \\
b_{21} & 0 & b_{23} & 0 \\
0 & -b_{14} & 0 & b_{34} \\
-b_{23} & 0 & b_{43} & 0
\end{array}\right)
$$

When waves propagate in the $x z\left(k_{y}=0\right)$ matrix elements takes the form:

$$
\begin{array}{lll}
b_{12}=i \varpi \mu_{0} \mu_{1} ; & b_{14}=-i \varpi \alpha_{\perp} ; & b_{21}=i \varepsilon_{0}\left(\frac{k_{x}^{2}}{\beta} \varepsilon_{2}+\omega \varepsilon_{1}\right) ; \\
b_{23}=-i\left(\frac{k_{x}^{2}}{\beta} \alpha_{11}+\omega \alpha_{\perp}\right) ; & b_{34}=-i \varpi \varepsilon_{0} \varepsilon_{1} ; & b_{43}=-i \mu_{0}\left(\frac{k_{x}^{2}}{\beta} \mu_{2}+\omega \mu_{1}\right) .
\end{array}
$$

When waves propagate in the $y z\left(k_{x}=0\right)$ matrix elements takes the form:

$$
b_{12}=i \mu_{0}\left(\frac{k_{y}^{2}}{\beta} \mu_{2}+\omega \mu_{1}\right) ; \quad b_{14}=-i\left(\frac{k_{y}^{2}}{\beta} \alpha_{11}+\omega \alpha_{\perp}\right) ; \quad b_{21}=i \varpi \varepsilon_{0} \varepsilon_{1}
$$




$$
b_{23}=-i \varpi \alpha_{\perp} ; \quad b_{34}=-i \varepsilon_{0}\left(\frac{k_{y}^{2}}{\beta} \varepsilon_{2}+\omega \varepsilon_{1}\right) ; \quad b_{12}=-i \varpi \mu_{0} \mu_{1} .
$$

The consequence of the matrix structure of coefficients of $\hat{B}$ is the structure of fundamental solutions:

$$
\hat{T}^{-1}=\left(\begin{array}{cccc}
t_{22} & -t_{12} & -t_{42} & -t_{32} \\
-t_{21} & t_{11} & t_{41} & -t_{31} \\
-t_{24} & t_{14} & t_{44} & -t_{34} \\
t_{23} & -t_{13} & -t_{43} & t_{33}
\end{array}\right) .
$$

Due to its wide application, inhomogeneous periodic medium is one of the important class of inhomogeneous medium. The structure of the fundamental solutions give the opportunity to find the most general dispersion equations of electromagnetic waves in inhomogeneous periodic medium with magnetoelectric effect.

When electromagnetic waves propagate in the coordinate planes dispersion equations are found from the following condition:

$$
\operatorname{det}(\hat{P}-\hat{E} \cos \tilde{k} h)=0,
$$

here

$$
\hat{P}=\frac{1}{2}\left(\hat{T}+\hat{T}^{-1}\right)
$$

From the structures of $T$ and $T^{-1}$ the structure of $P$ takes the form:

taking into account (12) in (10) gives:

$$
\hat{P}=\left(\begin{array}{cccc}
P_{11} & 0 & P_{13} & P_{14} \\
0 & P_{11} & P_{14} & P_{24} \\
-P_{24} & P_{14} & P_{33} & 0 \\
P_{14} & -P_{13} & 0 & P_{33}
\end{array}\right),
$$

$$
\tilde{P}_{1}, \tilde{P}_{2}=\frac{1}{2}\left(P_{11}+P_{22} \pm \sqrt{\left(P_{11}-P_{22}\right)^{2}+4\left(P_{14} P_{14}+P_{13} P_{24}\right)}\right),
$$

the general form of dispersion equations can be written in the following form:

$$
\cos \tilde{k}_{1} h=\tilde{P}_{1}, \cos \tilde{k}_{2} h=\tilde{P}_{2} .
$$

Averaged matricant, describing the propagation of electromagnetic wave in homogeneous anisotropic medium with magnetoelecric effect, is obtained in the following analytical form

$$
\hat{T}_{\text {aver }}^{ \pm}=\left(\hat{\pi}+\frac{1}{2} \hat{E}\right)\left(\hat{E} \cos k z \pm \frac{\hat{B}}{k} \sin k z\right)-\left(\hat{\pi}-\frac{1}{2} \hat{E}\right)\left(\hat{E} \cos \chi z \pm \frac{\hat{B}}{\chi} \sin \chi z\right)
$$

here

$$
\hat{\pi}=\frac{\hat{P}-\tilde{P}_{2} \hat{E}}{\tilde{P}_{1}-\tilde{P}_{2}}-\frac{1}{2} \hat{E} ; \hat{P}=\hat{E}+\frac{1}{2} \hat{B}^{2} h^{2} .
$$

Matriciants will have an appearance

where

$$
\begin{aligned}
& \hat{T}_{\text {aver }}^{+}=\left(\begin{array}{cccc}
t_{11} & t_{12} & t_{13} & t_{14} \\
t_{21} & t_{11} & t_{23} & t_{24} \\
-t_{24} & -t_{14} & t_{33} & t_{34} \\
-t_{23} & -t_{13} & t_{43} & t_{33}
\end{array}\right) ; \\
& \hat{T}_{\text {aver }}^{-}=\left(\begin{array}{cccc}
t_{11} & t_{12} & t_{13} & -t_{14} \\
t_{21} & t_{11} & -t_{23} & t_{24} \\
-t_{24} & t_{14} & t_{33} & t_{34} \\
t_{23} & -t_{13} & t_{43} & t_{33}
\end{array}\right),
\end{aligned}
$$




$$
\begin{aligned}
& t_{11}=\frac{\operatorname{Cos}[k z]\left(b_{12} b_{21}-b_{34} b_{43}+\Delta\right)}{2 \Delta} ; \\
& t_{12}=\frac{\operatorname{Sin}[k z]\left(b_{12}^{2} b_{21}-2 b_{14}^{2} b_{43}+b_{12}\left(-2 b_{14} b_{23}-b_{34} b_{43}+\Delta\right)\right)}{2 k \Delta} ; \\
& t_{13}=\frac{\operatorname{Cos}[k z]\left(b_{12} b_{23}+b_{14} b_{43}\right)}{2 \Delta} ; \\
& t_{14}=\frac{\operatorname{Sin}[k z]\left(b_{12}\left(b_{14} b_{21}+2 b_{23} b_{34}\right)+b_{14}\left(b_{34} b_{43}+\Delta\right)\right)}{2 k \Delta} ; \\
& t_{21}=\frac{\operatorname{Sin}[k z]\left(b_{12} b_{21}^{2}-2 b_{14} b_{21} b_{23}-b_{34}\left(2 b_{23}^{2}+b_{21} b_{43}\right)+b_{21} \Delta\right)}{2 k \Delta} ; \\
& t_{23}=\frac{\operatorname{Sin}[k z]\left(b_{12} b_{21} b_{23}+2 b_{14} b_{21} b_{43}+b_{23}\left(b_{34} b_{43}+\Delta\right)\right)}{2 k \Delta} ; \\
& t_{24}=\frac{\operatorname{Cos}[k z]\left(b_{14} b_{21}+b_{23} b_{31}\right)}{\Delta} ; \\
& t_{33}=\frac{\operatorname{Cos}[k z]\left(-b_{12} b_{21}+b_{34} b_{43}+\Delta\right)}{2 \Delta} ; \\
& t_{34}=\frac{\operatorname{Sin}[k z]\left(-2 b_{14}^{2} b_{21}-2 b_{14} b_{23} b_{34}+b_{34}\left(-b_{12} b_{21}+b_{34} b_{43}+\Delta\right)\right)}{2 k \Delta} ; \\
& t_{43}=\frac{\operatorname{Sin}[k z]\left(-b_{12}\left(2 b_{23}^{2}+b_{21} b_{43}\right)+b_{43}\left(-2 b_{14} b_{23}+b_{34} b_{43}+\Delta\right)\right)}{2 k \Delta},
\end{aligned}
$$

here

$$
\Delta=\sqrt{\left(b_{12} b_{21}-b_{34} b_{43}\right)^{2}-4\left(b_{12} b_{23}+b_{14} b_{43}\right)\left(b_{14} b_{21}+b_{23} b_{34}\right)}
$$

When $z=0$ matricant (15) can be written as:

$$
\hat{T}_{0}^{ \pm}=\frac{1}{2} \hat{E} \mp \hat{R}
$$

$\hat{R}$ matrix has the form:

$$
\hat{R}=\frac{1}{2 i}\left(\frac{k-\chi}{k \chi}\right) \pi \hat{B}-\frac{1}{4 i}\left(\frac{k+\chi}{k \chi}\right) \hat{B} .
$$

Assuming: $\vec{U}_{P}$ - field of incident waves, $\vec{U}_{R}$ - field of reflected waves and $\vec{U}_{t}-$ field of refracted waves, from (6) we have:

$$
T_{0}^{P} \vec{U}_{P}+T_{0}^{R} \vec{U}_{R}=T_{0}^{t} \vec{U}_{t}, \text { when } z=0 .
$$

Considering continuity of fields at the boundary:

$$
\vec{U}_{P}+\vec{U}_{R}=\vec{U}_{t}
$$

We get the result for reflected waves:

$$
\vec{U}_{R}=\left(R_{0}+\hat{R}_{t}\right)^{-1}\left(\hat{R}_{0}-\hat{R}_{t}\right) \vec{U}_{0} .
$$

Condition (19) with consideration of continuity of solutions at the boundary (20) is the matrix form of boundary conditions which are imposed on vectors of reflected, refracted and incident waves.

Then the fields of reflected and refracted waves:

$$
\begin{gathered}
\vec{U}_{R}=\hat{G} \vec{U}_{P} ; \\
\vec{U}_{t}=(\hat{G}+\hat{E}) \vec{U}_{P} .
\end{gathered}
$$

Analytical equations for homogeneous anisotropic medium with magnetoelectric effects allows one, in matrix setting, to obtain analytical solutions for the problem of reflection and refraction on the border of isotropic and anisotropic medium with magnetoelectric effect based on the matricant method. Initial relation- 
ships that describe electromagnetic wave propagation in anisotropic magnetoelectric medium are reduced to the system of linear homogeneous first order differential equations.

Using above algorithm, numerical calculations of energy flow density in the case of TE and TM incident waves at the boundary of two medium are conducted. The graphs of reflected energy coefficients when $\mathrm{TE}$ and TM electromagnetic waves are incident are plotted against incident angle.

\title{
References
}

1 Ландау Л.Д. Электодинамика сплошных сред / Л.Д. Ландау, Е.М. Лифшиц. — М.: Наука, 1982.

2 Тлеукенов С.К. Изучение электомагнитных полей в анизотропных средах / С.К. Тлеукенов, А.Т. Оспанов. - Алматы: Наука, 1985. - $176 \mathrm{c.}$

3 Тлеукенов С.К. О характеристической матрице периодически неоднородного слоя / С.К. Тлеукенов // Математические вопросы теории распространения волн. Т. 165. - Л.: Записки науч. семинара ЛОМИ, 1987. — С. $177-181$.

4 Тлеукенов С.К. Метод матрицанта / С.К. Тлеукенов. - Павлодар: НИЦ ПГУ им. С. Торайгырова, 2004. - 148 с.

5 Тлеукенов С.К. О методе решения некоторых задач распространения упругих волн при наличии периодической неоднородности. Т. 148 / С.К. Тлеукенов, О. Байгонысов. - Л.: Записки науч. семинара ЛОМИ АН СССР, 1985. - С. $30-33$.

6 Тлеукенов С.К. Электромагниттік толқындардың шағылу және сыну есептеріндегі шектік шарттардың матрицалық түрі / С.К. Тлеукенов, М.К. Жукенов, Н.К. Каратаева, Д. Жакипова // Вторые Ержановские чтения: материалы Междунар. науч. конф. - Актобе, 2007. - С. 278-281.

7 Тлеукенов С.К. К вопросу отражения электромагнитной ТЕ-волны от поверхности магнитоэлектрического кристалла гексагональной сингонии классов 622, 6mm, 6m2, 6mmm / С.К. Тлеукенов, М.К. Жукенов, Т.С. Досанов, Г.М. Жумабаева // Вестн. Караганд. ун-та. Сер. Физика. - 2016. - № 3. - С. 21-27.

8 Тлеукенов С.К. Метод матрицанта. Единое описание волновых процессов в средах с взаимной трансформацией упругих и электромагнитных полей / С.К. Тлеукенов, М.К. Жукенов // Вестн. Казах. нац. пед. ун-та. Сер. физ.-мат. наук. Алматы, 2016. - № 4. С. 85-91.

\author{
С.К. Тлеукенов, М.К. Жукенов, Н.А. Испулов
}

\section{Электрмагниттік толқындардың анизотропты магнитэлектрлік орталарда таралуы туралы}

\begin{abstract}
Мақалада магнитэлектрлік эффектісі бар тетрагоналды, тригоналды және гексагоналды сингониялы Z осі бойынша анизотропты орталарда поляризациясы әртүрлі электрмагниттік толқындардың таралуы мен әсерлесуі қарастырылды. Бастапқы теңдеулер жүйесінің матрицант құрылымы коэффициенттер матрицасының құрылымынан шығады. Шексіз периодты құрылымдардағы электрмагниттік толқындардың дисперсия теңдеулері матрицант құрылымының салдары болып табылатын жаңа модификацияланған шарттардан анықталады. Біртекті анизотропты магнитэлектрлік эффектісі бар диэлектрлік орталар үшін матрицанттардың анық аналитикалық түрі матрицант құрылымынан шығады. Магнитэлектрлік эффектісі бар біртекті анизотпропты орталар үшін аналитикалдық теңдеулер матрицалық түрде изотропты орта мен магнитэлектрлік эффектісі бар ортаның арасындағы шекарада толқындардың шағылу және сыну есептерін аналитикалық шешуіне мүмкіндік береді. Электрмагниттік толқындардың анизотропты магнитэлектрлік орталарда таралуын сипаттайтын бастапқы қатынастар сызықты біртекті бірінші ретті дифференциалдық теңдеулердің жүйесіне келтірілді. Матрицант құрылымы шығарылды. Біртексіз магнитэлектрлік эффектісі бар орталардағы электрмагниттік толқындардың дисперсия теңдеулері жазылды. Электрмагниттік толқындардың магнитэлектрлік эффектісі бар біртекті анизотропты орталарда таралуын сипаттайтын орташаланған матрицант құрылды. Энергиялық шағылу коэффициентінің электрмагниттік ТЕ және ТМ толқындардың түсу бұрышына тәуелділік графиктері салынды.
\end{abstract}

Кілт сөздер: анизотропты орталар, электрмагниттік толқындар, магнитэлектрлік эффект, матрицант әдісі, электрмагниттік толқындардың шағылуы, сынуы.

\section{С.К. Тлеукенов, М.К. Жукенов, Н.А. Испулов \\ О распространении электромагнитных волн в анизотропных магнитоэлектрических средах}

В статье распространение и взаимодействие электромагнитных волн различной поляризации в неоднородных вдоль оси Z анизотропных средах с магнитоэлектрическим эффектом тетрагональной, 
тригональной и гексагональной сингонии описываются структурой матрицы коэффициентов. Структура матрицанта исходной системы уравнений следует из структуры матрицы коэффициентов. В неограниченных периодических структурах уравнения дисперсии электромагнитных волн определяются из нового модифицированного условия существования нетривиальных решений, являющегося следствием структуры матрицанта. Явный аналитический вид матрицантов для однородных анизотропных диэлектрических сред с магнитоэлектрическим эффектом следует из структуры матрицанта. Аналитические формулы для однородных анизотропных сред с магнитоэлектрическим эффектом позволяют в матричной постановке получить аналитическое решение задачи отражения и преломления на границе изотропной среды и анизотропной среды с магнитоэлектрическим эффектом на основе метода матрицанта. Исходные соотношения, описывающие распространение электромагнитных волн в анизотропных магнитоэлектрических средах, приведены к системе линейных однородных дифференциальных уравнений первого порядка. Получена структура матрицанта. Построены уравнения дисперсии электромагнитных волн в периодически неоднородных средах с магнитоэлектрическим эффектом, усредненный матрицант, описывающий распространение электромагнитных волн в однородных анизотропных средах с магнитоэлектрическим эффектом. Кроме того, построены графики зависимости энергетического коэффициента отражения при падении электромагнитных ТЕ и ТМ волн от угла падения.

Ключевые слова: анизотропные среды, электромагнитные волны, магнитоэлектрический эффект, метод матрицанта, отражение, преломление электромагнитных волн.

\section{References}

1 Landau, L.D., \& Lifshitz, E.M. (1982). Elektrodinamika sploshnykh sred [Electrodynamics of continuous media]. Moscow: Nauka [in Russian].

2 Tleukenov, S.K., Ospanov, A.T. (1985). Izuchenie elektromahnitnykh polei v anizotropnykh sredakh [Investigation of electromagnetic fields in anisotropic medium]. Almaty: Nauka [in Kazakhstan].

3 Tleukenov, S.K. (1987). O kharakteristicheskoi matritse periodicheski neodnorodnoho sloia [On characteristic matrix of periodic inhomogeneous layer]. Matematicheskie voprosy teorii rasprostraneniia voln - Mathematical questions of wave propagation theory. Leningrad: Zapiski nauchnoho seminara LOMI AN SSSR [in Russian].

4 Tleukenov, S.K. (2004). Metod matritsanta [Matricant method]. Pavlodar: NITs PGU im. S. Toraighyrova [in Russian].

5 Baighonysov, O., \& Tleukenov, S.K.(1985) O metode resheniia nekotorykh zadach rasprosrtaneniia upruhikh voln pri nalichii periodicheskoi neodnorodnosti [On the method of solving some problems on propagation of elastic waves with the presence of periodic inhomogeneity]. Leningrad: Zapiski nauchnoho seminara LOMI AN SSSR, 30-33 [in Russian].

6 Tleukenov, S.K., Zhukenov, M.K., Karatayeva, N.K., \& Zhakipova, D. (2007) Electromahnittik tolkyndardyn shahylu zhane synu esepterindehi shektik sharttardyn matritsalyk turi [Matrix forms of boundary conditions in the problems of reflection and refraction of electromagnetic waves]. Proceedings from The second Erzhanov readings. Mezhdunarodnaia nauchnaia konferentsiia - International scientific conference, Aktobe, 278-281 [in Kazakh].

7 Tleukenov, S.K., Zhukenov, M.K., Dosanov, T.S., \& Zhumabayeva, G.M. (2016). K voprosu otrazheniia elekromahnitnoi TE-volny ot poverkhnosti mahnitoelekrticheskoho kristalla heksahonalnoi sinhonii klassov $622,6 \mathrm{~mm}, 6 \mathrm{~m} 2,6 \mathrm{mmm}[$ To an issue of reflection of an electromagnetic TE-wave from a surface of a magnetoelectric crystal of a hexagonal singoniya of classes $622,6 \mathrm{~mm}$, $6 \mathrm{~m} 2,6 \mathrm{mmm}$. Vestnik Karahandinskoho universiteta. Seriia Fizika - Bulletin of the Karaganda University. Physics series, 3, 21-27 [in Russian].

8 Tleukenov, S.K., \& Zhukenov, M.K. (2016). Metod matritsanta. Edinoe opisanie volnovykh protsessov v sredakh s vzaimnoi transformatsiei upruhikh i elektromahnitnykh polei [Matriciant method. The uniform description of wave processes in environments with mutual transformation of elastic and electromagnetic fields]. Vestnik Kazakhskoho natsionalnoho pedahohicheskoho universiteta. Seria fiziko-matematicheskikh nauk - Bulletin of the Kazakh National Pedagogical University. Physical and Mathematical sciences Series, 4, 85-91. 